\title{
Aprendizaje basado en proyectos en la asignatura de Metodología de Investigación en Educación Social
}

\author{
Juan Pablo Hernández Ramos
}

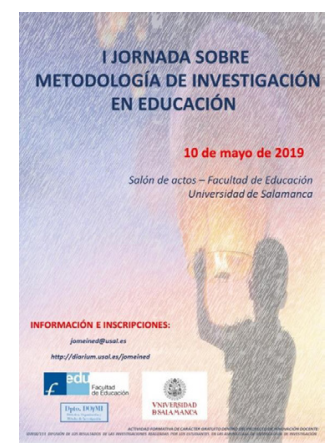

\section{$\mathrm{L}$} A Asignatura Metodología de investigación se encuentra presente en las titulaciones de la Facultad de Educación. En el Grado en Educación Social se ha aplicado esta asignatura.

Esta metodología coloca a los estudiantes como eje principal de su proceso de aprendizaje, encargándose del desarrollo de una investigación al completo, desde el planteamiento del problema hasta la difusión de resultados.

El beneficio formativo del empleo del ABP en esta asignatura se centra en la creación, procesamiento y difusión de conocimiento científico en la titulación de Educación Social.

Para su consecución se han planificado las siguientes acciones:

- Ofrecer un nexo entre la investigación educativa y la realidad social.

- Fomentar actividades de investigación en los estudiantes de Educación Social.

- Promover la difusión científica de resultados de investigación.

- Fomentar la difusión del conocimiento en abierto.

Con el empleo de la metodología ABP se pretenden desarrollar habilidades como la búsqueda, análisis, procesamiento y difusión de la información mediante el desarrollo de investigaciones científicas de rigor. 
Esta práctica acerca a los estudiantes a la realidad y les ofrece una motivación adicional. Se pretende que los estudiantes planifiquen, implementen y evalúen el mundo real, de manera que los aprendizajes lleguen fuera de las aulas. Es decir, que ofrezcan los resultados de sus investigaciones a la sociedad.

Este proyecto culmina en la realización de un acto público, que en este curso fue la I Jornada sobre Metodología de Investigación en Educación.

Por lo tanto, desde la selección de la temática de estudio hasta el día de la exposición pública del trabajo, los alumnos experimentan todas las fases de una investigación.

Los resultados de las investigaciones serán accesibles para toda la comunidad científica.

En cuanto a resultados del proyecto, se presentan dos perspectivas:

1. Los estudiantes: todos los alumnos cuyo trabajo fue

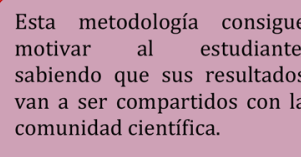

El objetivo es ofrecer a los estudiantes la posibilidad de aprender a investigar investigando la realidad.

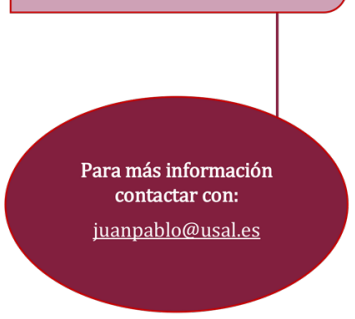
seleccionado para exponer en acto público, accedieron a participar, demostrando un alto grado de implicación propio de estudiantes comprometidos con la sociedad de forma participativa, lo que es el primer indicador de éxito.

2. La comunidad educativa: la jornada organizada les ha permitido conocer los resultados obtenidos en las investigaciones desarrolladas por los estudiantes gracias al empleo del ABP.

El carácter innovador reside en que la asignatura a la que nos referimos, una asignatura poco atractiva para los estudiantes, se convierte en una investigación completa que aborda todo el proceso de una investigación, aumentando la motivación y participación de los estudiantes, el interés sobre la materia e incluso las calificaciones.

En cuanto a la difusión de la práctica, se prevé la realización de, al menos, una publicación en revista científica sobre docencia universitaria.

De cara a la difusión de la práctica, se ha compartido la experiencia en diversos eventos y, con los trabajos de los estudiantes, se ha publicado la obra «Investigar en la sociedad, para la sociedad: Experiencias de los estudiantes» disponible en Gredos, el repositorio científico de la Universidad de Salamanca. 